\title{
Cutting edge data in autoimmunity: as presented in the 9th international congress of autoimmunity
}

\author{
Amir Dagan · Shaye Kivity
}

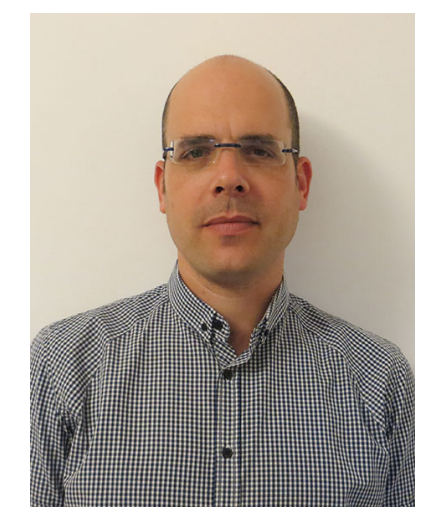

Amir Dagan

Published online: 4 December 2014

(C) Springer Science+Business Media New York 2014

In this issue of the cutting edge in autoimmunity, novelties in the fields of pathogenesis, diagnostics and clinical aspects of autoimmunity are presented to the readers. These innovative data were presented in the 9th International Congress of Autoimmunity held in Nice, France, earlier this year. The first part of the journal is dedicated to the ASIA syndrome-Autoimmune/inflammatory syndrome induced by adjuvants. This syndrome, recently described by Shoenlfeld and Agmon-Levin [1-3], relates to immunemediated symptoms triggered by an adjuvant stimulus. Pellegrino et al. reported the first epidemiological study, estimating the incidence of this syndrome using the vaccine adverse event reporting system (VAERS) database. This group estimates the incidence rate of ASIA following vaccination with HPV to be 3.6 cases per 100,000, thereby expanding our knowledge about the pathology of the syndrome. Butnato et al. deal with another aspect of the ASIA syndrome: the possible association between autoimmunity, adjuvants and malignancies. We already know that chronic inflammation triggered by infectious disease can lead to malignant processes e.g., H. Pylori and gastric cancer. We also know that connective tissue disease such as Sjogren's syndrome and systemic lupus erythematosus increase the risk of lymphoma $[4,5]$; can the chronic inflammatory process induced by adjuvants also increase the risk for lymphoproliferative diseases? The article by Butnato and

\footnotetext{
A. Dagan $(\bowtie) \cdot$ S. Kivity

Rheumatology Unit, The Zabludowicz Center for Autoimmune Diseases, Sheba Medical Center, Tel Hashomer, Israel

e-mail: Amir.Dagan@sheba.health.gov.il

S. Kivity

Sackler Faculty of Medicine, Tel Aviv University, Tel-Aviv, Israel
}

colleagues tries to look into this query from various perspectives.

Patients with adult onset still disease (AOSD) demonstrate extremely high ferritin levels. Hence, it was proposed that AOSD is part of the recently described 'hyperferritinemia syndrome' [6]. Jamilloux, in his review, explains several theories regarding the pathogenesis of AOSD. Ferritin has a major role in healthy subjects as one of the proteins responsible for iron regulation: It acts as a buffer against iron deficiency and iron overload. According to the study of Katz et al., we learn that not only ferritin but also $\beta$-carotene is involved in vitro in the regulation of iron in states of inflammation.

The involvement of the lung in autoimmune diseases is well known and extensively reported, with interstitial lung diseases (ILD) being one of the most common manifestations. Cryptogenic organizing pneumonia (COP), or as its former name bronchiolitis obliterans with organizing pneumonia (BOOP), is a histopathological pattern found in 6-16\% of RA-ILD [7]. However, in contrast to the relatively high prevalence of COP in RA, according to the article by Borella this type of lung involvement is only rarely part of the clinical picture in other connective tissue diseases (CTD's). In a literature review, they found only 32 case reports of BOOP linked to SLE, Sjogren's syndrome, systemic sclerosis, and inflammatory myopathies. Nevertheless, when BOOP does appear in CTD's, the course of the disease is more aggressive and less responsive to therapy than in idiopathic cases.

Vitamin D is an important mediator of innate immune responses, enhancing the antimicrobial properties of immune cells, such as monocytes, macrophages, and dendritic cells. Several studies reported an association between low levels of vitamin D and systemic lupus erythematosus $[8,9]$. Recently, we learnt about a link between vitamin $\mathrm{D}$ 
deficiency and the presence of antithyroid antibodies [10]. In this issue, Bizzaro et al. reviews the current knowledge regarding vitamin D deficiency and autoimmune thyroid disease. Among other subjects, she deals with the involvement of vitamin D in gestation of patients with Graves's disease. Bizzaro et al. proposes that vitamin D deficiency is linked to pregnancy loss as well as infertility in those patients. The autoimmunity aspects of pregnancy are further dealt with in two other articles in this journal: Bersani et al. evaluated the potential role of various cytokines, as possible biomarkers of pregnancy complicated by preeclampsia or chorioamnionitis in comparison with normal pregnancies. In this study, maternal serum interleukin6 (IL-6) levels were significantly higher in chorioamnionitis compared with preeclampsia and normal pregnancies, and fetal cord interleukin-22 (IL-22) levels were significantly higher in preeclampsia when compared with cord blood in chorioamnionitis and in normal pregnancies. IL-22 is a member of the IL-10 family cytokines which is produced by many different types of lymphocytes including both those of the innate and adaptive immune systems. Recently [11], accumulated evidence has indicated that IL-22, which generally acts as a proinflammatory cytokine, plays an important role in the pathogenesis of many autoimmune diseases. The increase in IL-22 levels witnessed by Bersani, without a similar rise in other proinflammatory cytokines i.e., IL-17, can suggest a different role for IL-22 in preeclampsia and should be further investigated. The second article by Tsur et al. deals with other aspects of the immune system on pregnancy. Former studies suggested that progesterone has anti-inflammatory properties mediated by suppression of Th- 1 and Th-17, as well as enhancement of regulatory $\mathrm{T}$ cell ( $\mathrm{T}$ regs) activity. Tsur et al. suggest that since preterm labor and recurrent pregnancy loss may be associated with inflammation, treatment with progesterone may quiescent the inflammatory process and maintain a healthy pregnancy. The authors further elaborate about the influence of progesterone and biological treatments on normal and abnormal pregnancies.

The diagnosis of autoimmune disease is largely based on the discovery of antibodies [12]. In the anti-phospholipid syndrome (APS), the presence of different antibodies is linked not only to the diagnosis but also to the severity of the syndrome. The anti-beta2-glycoprotein I (anti-ß2GPI) antibody is among the most clinically significant autoantibodies in APS. The $\beta 2$ GPI protein is composed of five domains and a subset of IgG anti- $\beta 2$ GPI directed against domain I on $\beta 2$ GPI seem to have a prominent role in the pathology of thrombotic complications in patients with APS [13]. Cucnic et al. examined the binding characteristics of patient's IgG anti- $\beta 2$ GPI to six $\beta 2 \mathrm{GPI}$-related peptide sequences corresponding to different domains of $\beta 2 \mathrm{GPI}$ and their association with diverse clinical manifestation of thrombosis. Another autoimmune disease whose classification is largely based on the presence of autoantibodies is rheumatoid arthritis (RA). Anti-cyclic citrullinated peptide (anti-CCP) and rheumatoid factor are used in clinical practice for diagnosis of RA, but are present in only $70-90 \%$ of the patients, mainly those with HLA-shared epitopes [14-16]. Recently, antibodies to carbamylated proteins (anti-carp) were described, and their presence in RA patients is associated with more severe joint damage, especially in anti-CCP negative patients [15]. In this journal, Yee et al. report a significant correlation between the presence of anti-carp antibodies and joint damage among 120 patients with rheumatoid arthritis.

Last but not least is a review by Esposito regarding the association between a rare inherited condition called epidermolysis bullosa and the mosaic of autoimmunity. This review displays in a nut shell the myriad aspects of the field of autoimmunity in this disease.

\section{References}

1. Shoenfeld Y, Agmon-Levin N. 'ASIA'—autoimmune/inflammatory syndrome induced by adjuvants. J Autoimmun. 2011;36(1):4-8.

2. Pludowski P, Holick MF, Pilz S, Wagner CL, Hollis BW, Grant WB, et al. Vitamin D effects on musculoskeletal health, immunity, autoimmunity, cardiovascular disease, cancer, fertility, pregnancy, dementia and mortality-a review of recent evidence. Autoimmun Rev. 2013;12(10):976-89.

3. Israeli F. Autoimimune/autoinflammatory syndrome induced by adjuvant (ASIA). Harefuah. 2014;153(7):397-400.

4. Bernatsky S, Ramsey-Goldman R, Labrecque J, Joseph L, Boivin $\mathrm{JF}$, Petri M, et al. Cancer risk in systemic lupus: an updated international multi-centre cohort study. J Autoimmun. 2013;42:130-5.

5. Tarella C, Gueli A, Ruella M, Cignetti A. Lymphocyte transformation and autoimmune disorders. Autoimmun Rev. 2013; 12(8):802-13.

6. Rosario C, Zandman-Goddard G, Meyron-Holtz EG, D’Cruz DP, Shoenfeld Y. The hyperferritinemic syndrome: macrophage activation syndrome, Still's disease, septic shock and catastrophic antiphospholipid syndrome. BMC Med. 2013;11:185.

7. Picchianti Diamanti A, Germano V, Bizzi E, Lagana B, Migliore A. Interstitial lung disease in rheumatoid arthritis in the era of biologics. Pulm Med. 2011;2011:931342.

8. Gatenby P, Lucas R, Swaminathan A. Vitamin D deficiency and risk for rheumatic diseases: an update. Curr Opin Rheumatol. 2013;25(2):184-91.

9. Cutolo M, Otsa K. Review: vitamin D, immunity and lupus. Lupus. 2008;17(1):6-10.

10. Kivity S, Agmon-Levin N, Zisappl M, Shapira Y, Nagy EV, Danko K, et al. Vitamin D and autoimmune thyroid diseases. Cell Mol Immunol. 2011;8(3):243-7.

11. Yang $X$, Zheng SG. Interleukin-22: a likely target for treatment of autoimmune diseases. Autoimmun Rev. 2014;13(6):615-20.

12. Tozzoli R, Villalta D. Autoantibody profiling of patients with antiphospholipid syndrome using an automated multiplexed immunoassay system. Autoimmun Rev. 2014;13(1):59-63.

13. Pengo V, Banzato A, Denas G, Jose SP, Bison E, Hoxha A, et al. Correct laboratory approach to APS diagnosis and monitoring. Autoimmun Rev. 2013;12(8):832-4. 
14. Pratesi F, Petit Teixeira E, Sidney J, Michou L, Puxeddu I, Sette A, et al. HLA shared epitope and ACPA: just a marker or an active player? Autoimmun Rev. 2013;12(12):1182-7.

15. Shi J, van Veelen PA, Mahler M, Janssen GM, Drijfhout JW, Huizinga TW, et al. Carbamylation and antibodies against carbamylated proteins in autoimmunity and other pathologies. Autoimmun Rev. 2014;13(3):225-30.

16. Sakkas LI, Bogdanos DP, Katsiari C, Platsoucas CD. Anti-citrullinated peptides as autoantigens in rheumatoid arthritis-relevance to treatment. Autoimmun Rev. 2014;13(11):1114-20. 